$\begin{array}{ll}\text { Revue d'Alsace } & \text { Revue d'Alsace } \\ & 133 \mid 2007 \\ & \text { Histoire régionale Landesgeschichte en France et en } \\ & \text { Allemagne 1950/2000 }\end{array}$

\title{
Une première étape !
}

François Igersheim et Jean-Pierre Kintz

\section{(2) OpenEdition \\ Journals}

Édition électronique

URL : http://journals.openedition.org/alsace/1445

DOI : $10.4000 /$ alsace. 1445

ISSN : 2260-2941

Éditeur

Fédération des Sociétés d'Histoire et d'Archéologie d'Alsace

Édition imprimée

Date de publication : 1 octobre 2007

Pagination : 7-8

ISSN : 0181-0448

\section{Référence électronique}

François Igersheim et Jean-Pierre Kintz, « Une première étape ! », Revue d'Alsace [En ligne], 133 | 2007, mis en ligne le 25 octobre 2011, consulté le 21 septembre 2020. URL : http://journals.openedition.org/ alsace/1445 ; DOI : https://doi.org/10.4000/alsace.1445 


\section{Une première étape!}

La Fédération des Sociétés d'Histoire et d'Archéologie d'Alsace est par nature productrice d'historiographie régionale. Elle en témoigne par ses grandes publications, ainsi le Nouveau Dictionnaire de Biographie Alsacienne et aussi par la Revue d'Alsace. Elle porte et fait connaitre la production des Sociétés d'histoire locale qu'elle fédère.

Elle n'est ni insulaire ni isolée, ni renfermée, ni exclusive, au contraire. Elle se sait et se veut porteuse d'un ferment de vitalité de la région Alsace, ancrée dans la conscience de ses habitants, c'est à dire qu'elle se veut ouverte aux quatre points cardinaux, s'inscrivant dans les meilleures traditions de l'historiographie alsacienne.

Lorsque Georges Livet et Christian Gras ont réuni à Strasbourg, le colloque de 1974, consacré à Régions et Régionalisme en France, l'idée régionale était à l'ordre du jour.

En 2006, elle y a pris racine ! Certaines régions de France ont conservé leurs fortes spécificités : l'on a voulu s'informer de l'état de leur recherche. Le caractère fédéral de l'Allemagne y avait maintenu vivantes les historiographies des Länder. Et tout comme les autres régions de France et comme les Länder de la RFA, la Région Alsace s'est ouverte à la coopération transfrontalière dans une Europe où les coopérations se nouent au dessus des frontières entre régions et universités!

Voilà qui a marqué le colloque réuni à Strasbourg dans la belle salle des Archives de la Communauté urbaine de Strasbourg où nous reçoit Mme Laurence Perry les 30 novembre, $1^{\text {er }}$ et 2 décembre 2006 et dont la Revue d'Alsace publie les contributions, sous une forme adaptée, dans les pages qui suivent.

Deux moments forts du colloque en témoignent éloquemment !

En premier lieu les allocutions de bienvenue qu'ont tenus devant nous, après notre hôte, le président de la Communauté Urbaine de Strasbourg, M. Robert Grossmann, le Président du Conseil régional d'Alsace, M. Adrien Zeller, aux côtés du Recteur d'Académie, M. Gérald Chaix, et Mme Anne Jacquemin, directrice de l'UFR des Sciences historiques, représentant le Président de l'Université Marc Bloch, que nous remercions très vivement de leur appui.

Et deuxièmement nombre de contributions qui insistent sur la nécessité de trouver des thématiques comparatives, qui dépassent la simple échelle de la région, qui dépassent les formules de coopération économique ou d'aménagement du territoire, mais s'attaquent franchement à l'examen comparatif de grandes questions qui préoccupent les historiens et tout particulièrement les historiens du temps présent!

Nous avons donc donné l'exemple ! C'est ainsi que la Fédération a pris toute sa place dans l'entreprise qui, à Strasbourg, a rassemblé un colloque pour faire le point sur l'historiographie régionale et sur la Landesgeschichte en France et en Allemagne depuis 1950.

Il suffit de les prendre l'une après l'autre pour voir la richesse des apports auxquels procèdent les contributions de tous les historiens que nous avons rassemblés, sur le plan de l'information réciproque, de la réflexion et de la critique ou des propositions. 
Nous les avons laissées dans l'ordre dans lequel elles avaient été faites lors du colloque. L'alternance d'exposés français et allemands permet d'établir une sorte de regard croisé qui renforce encore la démarche comparatiste.

Nous maintenons également la tradition de notre Revue, celle de publier des articles en français et en allemand (pourvus de résumés en français). Ce n'est pas au moment où en Bade-Wurtemberg on vote l'obligation de l'apprentissage du français en première langue que nous allons y déroger. Là aussi nous voulons donner l'exemple. Bref, face au constat qui se dégage de notre colloque de la nécessité d'un renouvellement de nos historiographies régionales, nous avons conscience de faire un premier pas, de prendre une initiative au niveau régional. Mais nous savons que ce n'est qu'une première étape. Un premier pas, qui doit être suivi d'autres. Nous faisons appel à tous nos partenaires de ce colloque pour prendre le relais.

François Igersheim

Professeur d'histoire d'Alsace à l'Université Marc Bloch
Jean-Pierre Kintz

Professeur émérite de l'Université Marc Bloch

Président de la Fédération des Sociétés d'histoire et d'archéologie d'Alsace

Ce numéro de la Revue d'Alsace titré "Histoire régionale/Landesgeschichte en France et en Allemagne " constitue la publication retravaillée des contributions apportées au colloque Historiographie régionale/Landesgeschichte en France et en Allemagne et ses vecteurs : Universités, Revues, Collections, Sociétés savantes, - Bilan critique du Second XXe siècle (1950-2000), tenu les 30 novembre, $1^{\text {er }}$ et 2 décembre 2006 aux Archives de la Ville et de la Communauté urbaine de Strasbourg dont nous tenons à remercier la direction et le personnel. Il a été organisé en commun par la Fédération des sociétés d'histoire et d'archéologie d'Alsace, par l'Institut d'Histoire de l'Alsace (l'UFR des Sciences historiques de l'Université Marc Bloch), le groupe espaces rhénans et germaniques de l'EA 3400 de l'Université Marc Bloch et l'Université Marc Bloch de Strasbourg. Nous remercions l'Office pour la Langue et la Culture d'Alsace et la Région Alsace qui nous ont apporté leur aide. 